\title{
Interpreting the Consequences of Metformin Accumulation in an Emergency Context: Impact of the Time Frame on the Blood Metformin Levels
}

\author{
Jean-Daniel Lalau ${ }^{1,2}$ and Farshad Kajbaf ${ }^{2}$ \\ ${ }^{1}$ Service d'Endocrinologie-Nutrition, Hôpital Sud, 80054 Amiens, France \\ ${ }^{2}$ INSERM U-1088, Université de Picardie Jules Verne, 80037 Amiens, France \\ Correspondence should be addressed to Jean-Daniel Lalau; lalau.jean-daniel@chu-amiens.fr
}

Received 7 October 2014; Accepted 22 November 2014; Published 17 December 2014

Academic Editor: Mario Maggi

Copyright (C) 2014 J.-D. Lalau and F. Kajbaf. This is an open access article distributed under the Creative Commons Attribution License, which permits unrestricted use, distribution, and reproduction in any medium, provided the original work is properly cited.

Objective. To clarify the link between metformin accumulation and its metabolic consequences by taking the time frame for metformin measurement into account. Research Design and Methods. Our database was studied for cases of metformin accumulation and lactic acidosis status available on admission, and then we selected patients in whom arterial $\mathrm{pH}$, blood lactate, and plasma and erythrocyte metformin levels had been determined at the same time point. Results. Seventeen reports were studied on 16 patients, of whom 10 presented lactic acidosis. The time interval between admission and comprehensive testing ranged from 0 to 52 hours. The study parameters were determined simultaneously on admission in only 4 patients. In the 9 patients with lactic acidosis on admission and a delayed metformin assay, lactic acidosis persisted in 6 cases and had resolved in 3 cases by the time the blood sampling for metformin assay was performed. Conversely, lactic acidosis developed after admission in one case. Conclusions. Caution must be taken when interpreting the consequences of metformin accumulation in an emergency context: the patient's lactic acidosis status will have changed by the time the metformin assay is performed, even though metformin accumulation may still be present.

\section{Introduction}

It is well known that there is a relationship between metformin levels and lactate metabolism: the higher the blood metformin concentration, the more severe the metabolic disturbance $[1,2]$. However, the relationship between metformin and lactic acidosis is more complex because (i) lactic acidosis is not necessarily accompanied by metformin accumulation in metformin-treated patients; (ii) metformin accumulation does not necessarily lead to lactic acidosis; and, conversely, (iii) metformin may induce lactic acidosis in the absence of drug accumulation [3].

Studying the relationship between metformin and lactic acidosis therefore involves taking account of putative confounding factors [4], such as the patient profile, the disease context, the assay methods, the type of metformin accumulation (acute versus chronic), the time of the last metformin administration, the large interindividual variations in blood metformin levels for a given therapeutic dose, and, lastly, interindividual variations in the metabolic response to metformin accumulation $[5,6]$. Surprisingly, the time frame for determining the status of metformin accumulation has not previously been considered as a confounding factor, even though a delay in measuring blood metformin levels is largely unavoidable in an emergency context in which a metformin assay is not the top priority. Indeed, the late performance of a blood metformin assay may underestimate the level of drug present in the patient on admission (which should be followed by metformin's withdrawal as a result of emergency admission), especially when the patient is then treated with dialysis and/or the administration of vasoactive drug. Furthermore, the latter procedures may also rapidly modify the blood lactate concentration.

We therefore sought to clarify the link between metformin accumulation and its metabolic consequences by taking the time frame for metformin measurement into 
account and test the following hypothesis: the patient's lactic acidosis status will have changed by the time the metformin assay is performed.

Accordingly, we recorded (i) the proportion of patients for whom the metformin assay was delayed after their admission; (ii) the mean (range) of the time interval between the determination of lactic acidosis on admission and the metformin measurement; and (iii) the impact of the time interval between these two determinations on the assessment of the patient's metabolic status.

\section{Research Design and Methods}

2.1. Data Source. We systematically reviewed all the blood metformin assay data recorded by our hospital's laboratory between January 2004 and December 2013. In general, metformin assays had been requested in order to adjust the dose to the patient's renal status or to screen metformin accumulation. We then selected for analysis all patients with metformin accumulation (defined as a plasma metformin concentrations $\geq 5 \mathrm{mg} / \mathrm{L}$, corresponding to 10 times the mean reported therapeutic value [7]) and with available lactic acidosis status on admission.

2.2. Selection and Presentation of the Study Parameters. On the basis of the reports on patients with metformin accumulation, we noted the $\mathrm{pH}$, lactate, and the plasma and erythrocyte metformin concentrations. We selected patients in whom all these parameters had been determined at the same time point during hospitalization. The data were then analysed for (i) lactic acidosis (arterial $\mathrm{pH}<7.35$ and a blood lactate concentration $>5 \mathrm{mmol} / \mathrm{L}$ [8]) on admission and the time of the metformin assay and (ii) the extent of metformin accumulation.

\section{Results}

We identified 17 eligible reports on 16 patients aged between 44 and 74 . For each individual, the clinical setting, arterial $\mathrm{pH}$, arterial lactate, serum creatinine and blood metformin values, and the time interval between admission and subsequent assays are presented in Table 1 (cases 5 and 9 concern two different episodes in the same patient). All patients had hyperlactatemia (with a minimal value of $3.3 \mathrm{mmol} / \mathrm{L}$ ) and 10 patients had lactic acidosis. At least one factor for hyperlactatemia associated with metformin accumulation was identified in all but one case. Kidney disease and sepsis appeared to be the main factors associated with metformin accumulation. The serum creatinine concentration was available on admission for 14 cases and was abnormal in 13 of them. Dialysis and/or the administration of vasoactive drugs were initiated prior to blood metformin determination in four and six patients, respectively.

Arterial $\mathrm{pH}$ and lactate were available for all 16 patients on admission. Eleven of the latter had acidosis. The study parameters were determined simultaneously on admission in only 4 patients. The time interval between the assessment of lactic acidosis status on admission and sample collection for the full set of parameters varied greatly from one patient to another; it was between 0 and 6 hours in eight cases, between 6 and 12 hours in five cases, between 12 and 24 hours in one case, between 1 and 2 days in two cases, and over 3 days (52 hours, in fact) in one case. The mean time interval was $12.5 \pm 16$ hours. In the 9 cases with lactic acidosis on admission and for whom the sampling for the metformin measurement was performed later, lactic acidosis persisted at the time of such measurement in 6 cases and had resolved in 3 cases. Conversely, one patient (case 9) developed lactic acidosis after admission.

Blood metformin levels were high in all reports, even after dialysis and even for the longest time to blood sampling, and ranged from 6.3 to $66.8 \mathrm{mg} / \mathrm{L}$ in plasma (mean \pm SD: $28.7 \pm$ 21.3; normal upper limit $<1.35$ ) and 1.5 to $20.1 \mathrm{mg} / \mathrm{L}$ in erythrocytes (mean \pm SD: $12.3 \pm 6.6$; normal upper limit $<1.65$ ). The plasma metformin levels were higher than the erythrocyte metformin levels whenever the time to blood sampling after admission was below 8 hours and in half of the cases with a longer time interval.

Lastly, three of the 16 patients (18.8\%) failed to survive.

\section{Discussion}

The present study is the first to document patients with marked metformin accumulation as a function of the time frame for blood sampling. Lactic acidosis was observed in 10 out of the 17 reports in our series.

In view of the issues highlighted in the Introduction, the following observations may be made: (i) in most cases (13 out of 17 ), the $\mathrm{pH}$, lactate, and metformin values were not determined simultaneously on admission; (ii) the time interval between the initial sampling (for $\mathrm{pH}$ and lactate) and the subsequent sampling for obtaining other study parameters (including the metformin level) was generally rather short (about 12 hours) but varied markedly when considered the SD (16 hours) and especially the range (up to 52 hours); most importantly, (iii) the lactic acidosis status (i.e., resolution or occurrence) had changed in half of the patients for whom blood metformin levels had been determined with some delay.

It is also noteworthy that the three patients with the longest time interval between admission and subsequent sampling (about two days) still had very high plasma and erythrocyte metformin levels. At the time of the metformin measurement, the $\mathrm{pH}$ and lactate values no longer met the criteria for lactic acidosis. Although a high erythrocyte metformin concentration was expected (because of the drug's long half-life in these cells [9]), the persistent, high plasma concentration is surprising, especially since dialysis had been initiated in two of the three patients.

This study shares the limitations of all descriptive studies. However, it is fair to say that a trial limitation may be strength when it reflects what actually happens in clinical practice. More precisely, the present study's main objective was not to rigorously study the effect of metformin accumulation on lactate metabolism but rather to examine possible pitfalls when considering the link between metformin and so-called "metformin-associated lactic acidosis" under emergency 


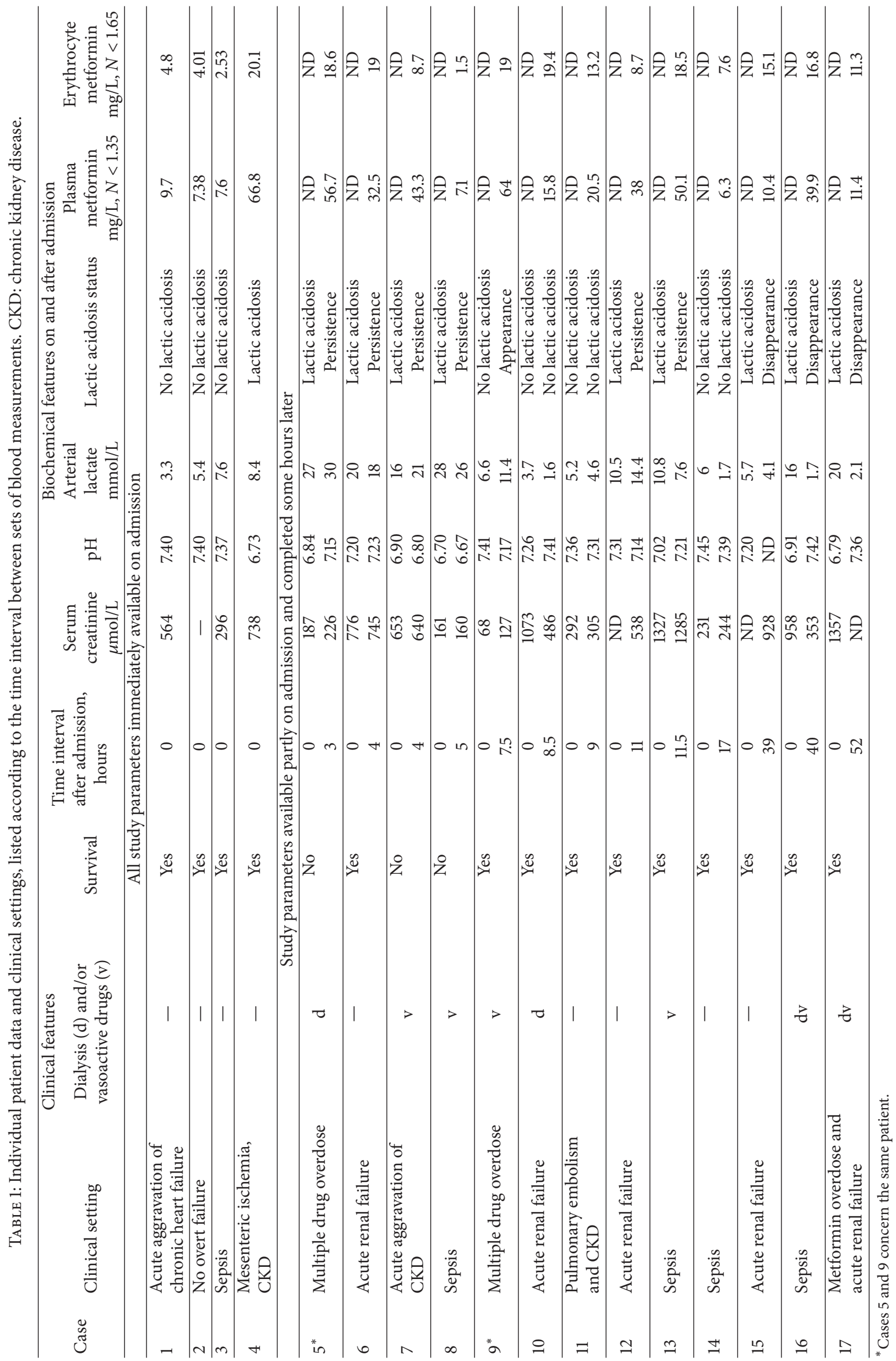


conditions. In other words, we wanted to know which factors have an effect on this link, such as the time frame for blood sampling for $\mathrm{pH}$, lactate, and metformin measurements.

There are two levels of complexity in this context: factors that modify the metformin level (such as the time to sampling after admission, in particular) and the physiopathological mechanism complexity of so-called "metformin-associated lactic acidosis" in itself [3]. Indeed, metformin accumulation may be related to either acute, primary renal failure or isolated metformin intoxication [10] (which occurred in less than one-third of the patients studied here) or secondary to renal failure as a complication of a severe, systemic disease (such as sepsis and heart failure) [11, 12]. For this reason, the existence of the term "metformin-associated lactic acidosis" does not imply that all the associated factors (e.g., the degree of metformin accumulation and the severity of any associated diseases) are present to the same degree at any given time.

Given the above results and considerations, our results suggest that when the time interval between the first lactate measurement and that of blood metformin is very long, caution must be taken when considering the putative relationship between metformin accumulation and lactic acidosis. Indeed, the patient's lactic acidosis status will have changed in some cases by the time the metformin assay is performed, even though metformin accumulation may still be present.

\section{Conclusion}

In patients with metformin accumulation, metformin levels are generally measured many hours after admission. Because of this delay, the patient's metabolic status (i.e., the presence or the absence of lactic acidosis) had changed in half of the cases studied here, even while metformin accumulation persisted. This delay should be taken into consideration when classifying patient with so-called "metformin-associated lactic acidosis."

\section{Conflict of Interests}

Jean-Daniel Lalau and Farshad Kajbaf declare that they have no conflict of interests.

\section{Acknowledgments}

Jean-Daniel Lalau and Farshad Kajbaf both contributed to conception, design, acquisition of data or analysis, and interpretation of data; Jean-Daniel Lalau was involved in drafting and revising the paper. Both authors read and approved the final paper. The present study was approved by the local investigational review board (Commission d'Evaluation Ethique des Recherches Non Interventionnelles, Espace Ethique Hospitalier Amiens Picardie, Amiens, France; ref.: 2014-4).

\section{References}

[1] M. Prikis, E. L. Mesler, V. L. Hood, and W. J. Weise, "When a friend can become an enemy! Recognition and management of metformin-associated lactic acidosis," Kidney International, vol. 72, no. 9, pp. 1157-1160, 2007.

[2] N. Peters, N. Jay, D. Barraud et al., "Metformin-associated lactic acidosis in an intensive care unit," Critical Care, vol. 12, no. 6, article R149, 2008.

[3] J.-D. Lalau, P. Arnouts, A. Sharif, and M. E. de Broe, "Metformin and other antidiabetic agents in renal failure patients," Kidney International, 2014.

[4] M. R. Gionfriddo, O. L. Morey-Vargas, J. P. Brito, A. L. Leppin, M. H. Murad, and V. M. Montori, "Systematic reviews to ascertain the safety of diabetes medications topical collection on pharmacologic treatment of type 2 diabetes," Current Diabetes Reports, vol. 14, article 478, 2014.

[5] M. M. Christensen, C. Brasch-Andersen, H. Green et al., "The pharmacogenetics of metformin and its impact on plasma metformin steady-state levels and glycosylated hemoglobin Alc," Pharmacogenetics and Genomics, vol. 21, no. 12, pp. 837-850, 2011.

[6] F. Robert, S. Fendri, L. Hary, C. Lacroix, M. Andréjak, and J. D. Lalau, "Kinetics of plasma and erythrocyte metformin after acute administration in healthy subjects," Diabetes and Metabolism, vol. 29, no. 3, pp. 279-283, 2003.

[7] J.-D. Lalau, A.-S. Lemaire-Hurtel, and C. Lacroix, "Establishment of a database of metformin plasma concentrations and erythrocyte levels in normal and emergency situations," Clinical Drug Investigation, vol. 31, no. 6, pp. 435-438, 2011.

[8] D. Luft, G. Deichsel, R. M. Schmulling, W. Stein, and M. Eggstein, "Definition of clinically relevant lactic acidosis in patients with internal diseases," The American Journal of Clinical Pathology, vol. 80, no. 4, pp. 484-489, 1983.

[9] J.-D. Lalau and C. Lacroix, "Measurement of metformin concentration in erythrocytes: Clinical implications," Diabetes, Obesity and Metabolism, vol. 5, no. 2, pp. 92-98, 2003.

[10] S. Vecchio and A. Protti, "Metformin-induced lactic acidosis: no one left behind," Critical Care, vol. 15, article 107, 2011.

[11] B. A. Mizock and J. L. Falk, "Lactic acidosis in critical illness," Critical Care Medicine, vol. 20, no. 1, pp. 80-93, 1992.

[12] F. Kajbaf and J.-D. Lalau, “The prognostic value of blood $\mathrm{pH}$ and lactate and metformin concentrations in severe metforminassociated lactic acidosis," BMC Pharmacology and Toxicology, vol. 14, article 22, 2013. 


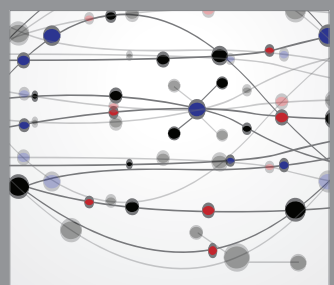

The Scientific World Journal
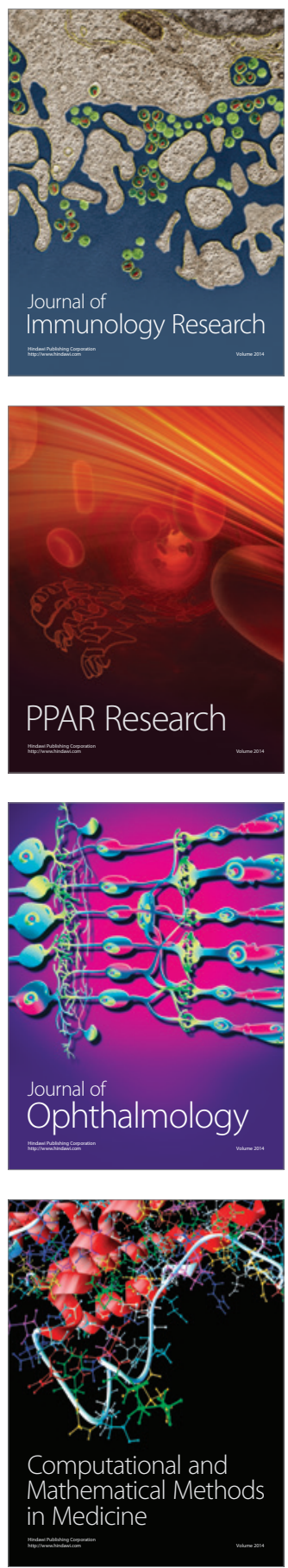

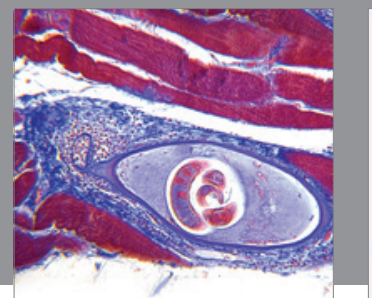

Gastroenterology

Research and Practice
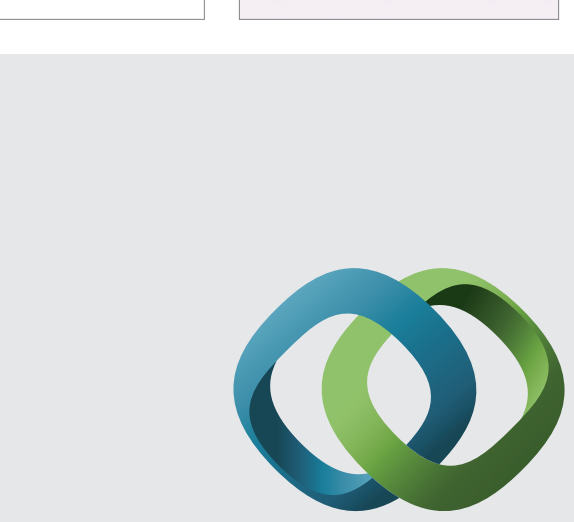

\section{Hindawi}

Submit your manuscripts at

http://www.hindawi.com
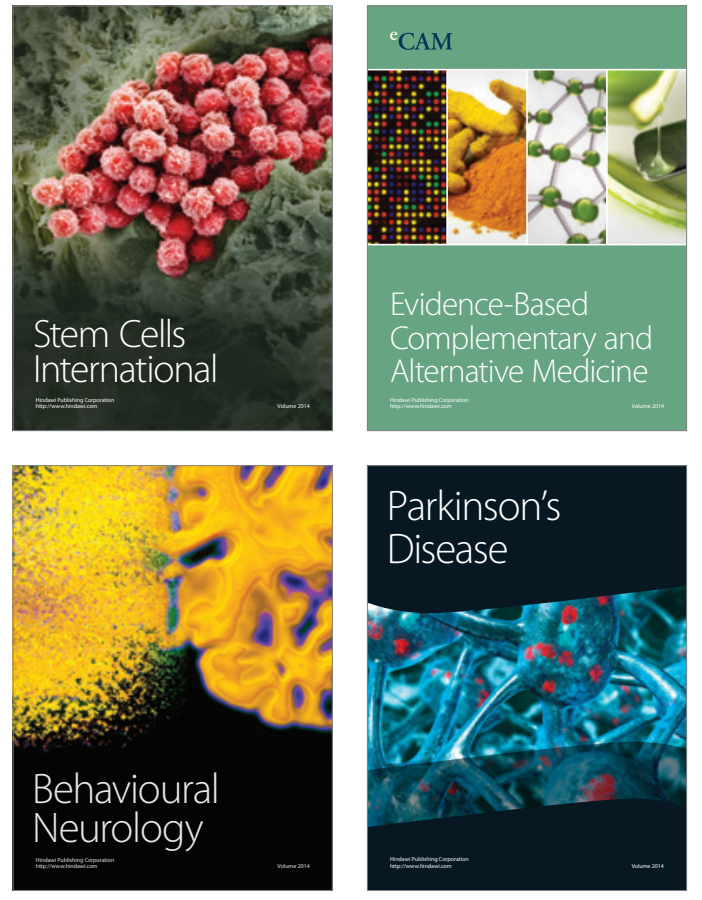
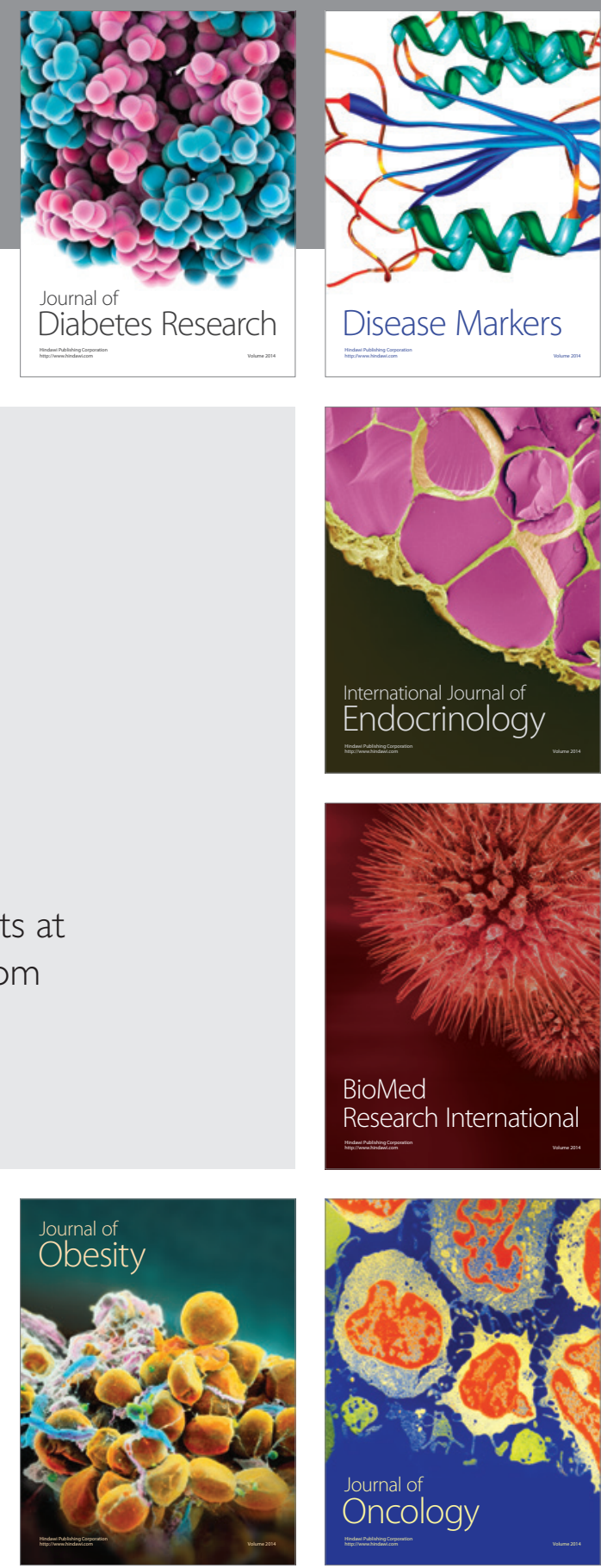

Disease Markers
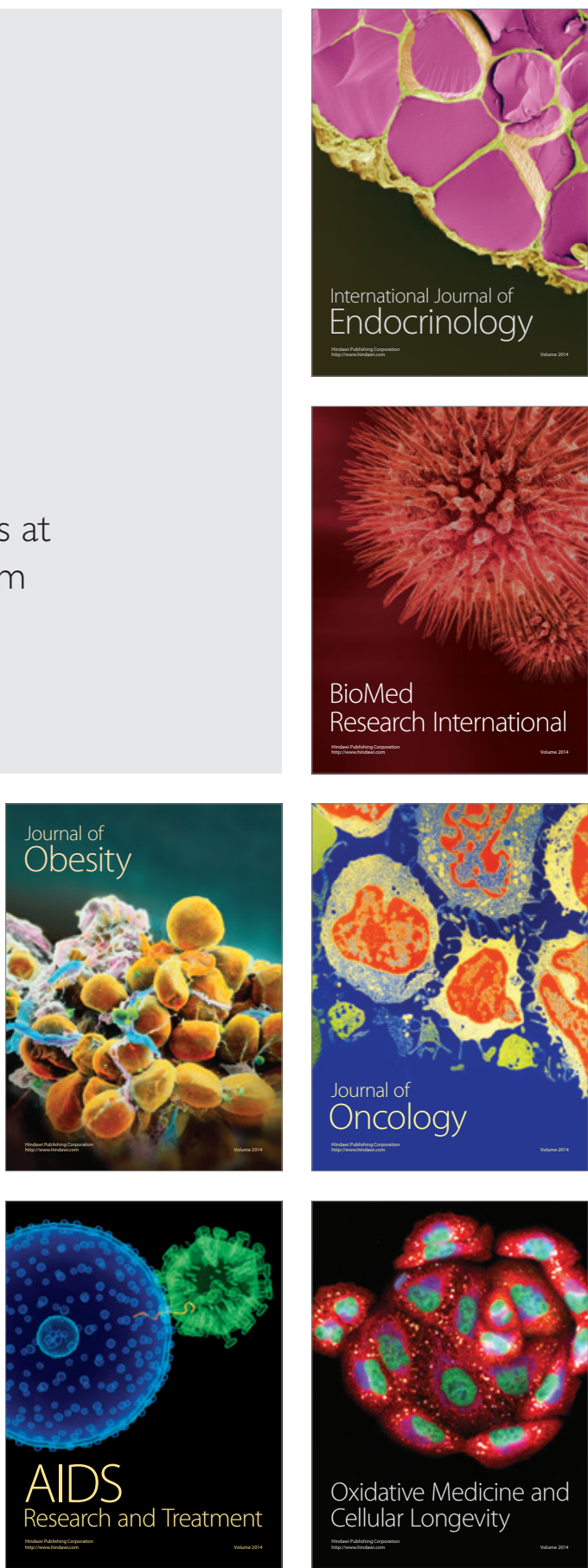